\title{
Solar Water Splitting: Photocatalyst Materials Discovery and Systems Development
}

Thomas F McNulty (Primary Contact)

GE Global Research

1 Research Circle

Niskayuna, NY 12309

(518)387-4108, Fax: (518)387-7563, mcnulty@research.ge.com

DOE Technology Development Manager: Roxanne Garland

(202)586-7260, Roxanne.Garland@hq.doe.gov

DOE Project Officer: David Peterson

(303)275-4956,david.peterson@go.doe.gov

Contract Number: DE-FG36-05GO15024

Subcontractors:

California Institute of Technology

Pasadena, CA 91125

Start Date: 07/15/2005

End Date: 01/30/2008 


\section{Executive Summary}

Hydrogen promises to be an attractive transportation fuel in the post-fossil fuel era. Relatively abundant and clean burning (water being the principal byproduct), hydrogen offers the potential to significantly reduce greenhouse gas emissions. However, there are significant technical barriers that require solutions before hydrogen can be implemented in large scale. These are:

- Sources (e.g. hydrocarbon, water)

- Transportation

- Storage

Each of the aforementioned barriers carries with it important considerations. First, would a hydrocarbonbased hydrogen source be of any benefit compared to conventional fossil fuels? Second, will a system based on centralized generation and distribution be viable? Finally, methods of on-board storage, whether they are liquefaction, adsorption, or intercalation, are far from optimized.

The scope of this program is limited to hydrogen generation, specifically generation using solarinitiated water electrolysis. Though concept of making hydrogen using water and sunlight may sound somewhat far-fetched, in reality the concept is very real. Since the discovery of solar-generated hydrogen, termed photoelectrochemical hydrogen, nearly 30 years ago by Fujishima and Honda, significant advances in both fundamental understanding and technological capability have been made ${ }^{1,2,3}$. Using solar radiation to generate hydrogen in a fashion akin to using solar to generate electricity offers many advantages. First, hydrogen can be generated at the point of use, reducing the importance of transportation. Second, using water as the hydrogen source eliminates greenhouse gas evolution and the consequences that come with it. Finally, because the process uses very little electricity (pumps and compressors predominantly), the quantity of chemical fuel produced far exceeds the amount of electricity consumed. Consequently, there is some level of truth to the notion that photoelectrochemically-derived hydrogen offers the potential to nearly eliminate greenhouse gas emissions from the transportation landscape.

This report focuses primarily on the technical issues inherent to developing an economically viable photoelectrochemical hydrogen system. This involves research intended to address technology gaps as well as research to address commercial feasibility. Though a firm cost target is not identified explicitly, much of the economics are presented in terms of "dollars per gallon of gasoline equivalent" (\$/gge). Obviously this is a moving target, but it is important to understand cost in terms of current gasoline pricing, since the intended target is gasoline replacement. However, this does put the cost contribution into a perspective that at least allows for a reasonable assessment of technological viability. It also allows for the identification of need areas beyond the obvious technology gaps.

\footnotetext{
${ }^{1}$ Fujishima et al, Nature, 238 (1972) 37-38

${ }^{2}$ Gratzel, Nature, 414 (2001) 338-344

${ }^{3}$ Khaselev et al, Science, 280 (1998) 425-427
} 


\section{Background}

Photoelectrochemical processes are ones that utilize the energy contained in the sun's incident radiation to carry out chemical reactions. Photosynthesis is of course the most familiar of this general family of reactions. For the case of water decomposition, the general reaction can be represented as ${ }^{4}$ :

$$
2 h v+\mathrm{H}_{2} \mathrm{O} \rightarrow \mathrm{H}_{2}+1 / 2 \mathrm{O}_{2}
$$

Where:

$$
h v=\Delta \mathrm{G} / 2 \mathrm{~N}_{\mathrm{A}}
$$

The Gibbs free energy for water is $237 \mathrm{~kJ} / \mathrm{mol}$, meaning the minimum photon energy required to initiate photoelectrochmically-induced water is:

$$
h v=\left(237 \times 10^{3} \mathrm{~J} / \mathrm{mol}\right) / 2 \cdot\left(6.023 \times 10^{23} \text { electrons } / \mathrm{mol}\right) /\left(1.6 \times 10^{-19} \mathrm{eV} / \mathrm{mol}\right)=1.23 \mathrm{eV}
$$

Consequently, photons with energy greater than 1.23 electron volts contain enough energy to split the water molecule.

Sufficient energy is of course not the only requirement; otherwise solar radiation incident on pools of water would readily create hydrogen. How the energy is delivered to the molecule matters.

Fundamentally, oxidation of a water molecule and reduction of two hydrogen ions takes place

simultaneously. For a photoelectrochemical system such as the one discussed here, this requires the generation of an electrochemical potential across an anode and cathode immersed in an electrolyte, efficient transfer of two electrons from a water molecule to the anode, transfer of electrons from the anode to the cathode, and subsequent transfer of two electrons from the cathode to two hydrogen ions. This is shown diagrammatically in Figure 1. As described by in Ref. 4, there are 3 arrangements that satisfy the aforementioned conditions:

1. Photo-anode made of n-type semiconductor and cathode made of metal

2. Photo-anode made of n-type semiconductor and photo-cathode made of p-type semiconductor

3. Anode made of metal and photo-cathode made of p-type semiconductor

There are critical differences between the three arrangements that require further discussion. For case 1, the oxidation reaction takes place on the surface of the semiconductor. This is of particular importance, especially for non-oxide photo-anode such as those studied in this work, since this opens the possibility of photo-induced oxidation of the photo-anode. For case 2, the aforementioned photo-induced degradation is also possible, however the presence of two photo-catalysts allows for increased design and materials flexibility not possible in single photo-catalyst systems. This comes at a cost to efficiency due to the fact that photo-anode / photo-cathode "tandem" devices require four photons to complete the reaction rather than two. In case 3, the reduction reaction takes place on the semiconductor surface, which is more desirable in the case of non-oxide photo-catalysts.

Thus far, we've identified five conditions that are necessary for efficient hydrogen production. These are:

1. Efficient absorption of photons with energy greater than $1.23 \mathrm{eV}$.

2. Generation of an electrical potential between an anode and cathode.

3. Efficient transfer of two electrons from a water molecule to the anode.

4. Current flow from the anode to the cathode of the photoelectrochemical system.

5. Efficient transfer of two electrons from the cathode to hydrogen ions in solution with water.

\footnotetext{
${ }^{4}$ Bak et al, Int. J. Hydrogen Energy, 27 (2002) 991-1022
} 
One final condition yet to be discussed is the requirement that the band energy levels of the photoelectrochemical cell (PEC) are required to straddle the oxidation and reduction potentials of water and hydrogen, respectively. For the simple PEC system shown in Figure 1, this means that not only the valence and conduction bands of the photo-anode, but also the Fermi level of the cathode. Furthermore, the $\mathrm{pH}$ of the electrolyte determines the energy levels of the $\mathrm{H}^{+} / \mathrm{H}_{2}$ and $\mathrm{H}_{2} \mathrm{O} / \mathrm{O}_{2}$ redox potentials. This is shown in Figure 2.

Taken together, the aforementioned conditions put considerable constraints on the design and construction of a photoelectrochemical system. Holistically, this is a considerable challenge. However, by focusing on individual aspects of the problem, considerable progress towards a commercially robust PEC system is possible. This project addresses the following technical barriers identified for photoelectrochemical hydrogen systems from Section 3.1 of the Hydrogen, Fuel Cells and Infrastructure Technologies Program Multi-Year Research, Development and Demonstration Plan:
1. Y. Materials Efficiency
2. Z. Materials Durability
3. AB. Bulk Materials Synthesis
4. AC. Device Configuration Designs
5. AD. Systems Design and Evaluation

In particular, materials issues related to the discovery, development, and optimization of an efficient and durable photo-catalyst is investigated. Furthermore, device configuration designs were carried out, targeting the system simplicity required to achieve parity with conventional fossil fuel transportation fuels.

\section{Technical Targets}

This program targets the development of photoelectrochemical systems with an installed system cost and service life capable of achieving DOE's long-term target of gasoline parity for a hydrogen transportation fuel. The system level technical targets are outlined in Table 3.1.10 of the 2007 DOE Hydrogen Plan. In particular, this program targets the development of photo-catalysts capable of $>12 \%$ solar-to-hydrogen (STH) efficiency. Furthermore, materials with sufficient durability to exceed the targeted 5000-hour continuous operation will be investigated. Finally, low-cost device configurations with simple and robust architecture will be investigated.

\section{Approach}

\section{Design Considerations:}

Calculations to determine entitlement are helpful in gaining an appreciation for the performance expectations. First, at $0.00202 \mathrm{~kg} / \mathrm{mol}$, there are roughly 495 moles of hydrogen per kilogram. Second, since there are 2 electrons required for the formation of one molecule of hydrogen, we find:

$$
(2 \mathrm{e}-/ \text { molecule }) \cdot\left(495 \mathrm{~mol}-\mathrm{H}_{2} / \mathrm{kg}\right) \cdot(96485 \mathrm{C} / \mathrm{mol})=9.55 \times 10^{7} \mathrm{C} / \mathrm{kg}-\mathrm{H}_{2}
$$

Assuming 2015 targets of $12 \%$ chemical conversion efficiency; $2.0 \mathrm{eV}$ bandgap, we find for the city of Phoenix $\left(250 \mathrm{~W} / \mathrm{m}^{2}\right.$ average insolation):

$$
\left(250 \mathrm{~W} / \mathrm{m}^{2}\right) \cdot(0.12) /(2.0 \mathrm{~V})=15 \mathrm{~A} / \mathrm{m}^{2}=15 \mathrm{C} / \mathrm{m}^{2} / \mathrm{sec}=1,296,000 \mathrm{C} / \mathrm{m}^{2} / \text { day }
$$

Thus, to produce $1 \mathrm{~kg}$ of hydrogen, a $1 \mathrm{~m}^{2}$ panel would require nearly 73 days. Clearly, this puts the challenge into perspective, particularly with respect to system cost. The average refueling station in the US will sell about 2000 gallons of fuel every day. As mentioned earlier, this would correspond to $2000 \mathrm{~kg} / \mathrm{day}$ of hydrogen. However, if one takes into account the anticipated doubling of energy efficiency due to widespread use of hydrogen fuel cell vehicles, $1000 \mathrm{~kg} /$ day could be more realistic. For this kind of output, that would require roughly $73,000 \mathrm{~m}^{2}$ of PEC cell area, or roughly four average big-box store rooftops.

The size of PEC systems will require changes in where and how people refuel their vehicles. This is largely a consequence of the fact that relatively speaking, the solar energy flux is not particularly intense. 
For comparison, an alkaline electrolyzer typically operates at about $1500 \mathrm{~A} / \mathrm{m}^{2}$, compared to a PEC system which is expected to operate at a current flux roughly two orders of magnitude lower.

One final consideration has to do with the seasonal variability of hydrogen generation, particularly in locations with significant seasonal variability in total sunlight. As shown in Table 1, regions such as the northern US and Canada fall into this category. Consequently, exclusive implementation of a PEC system of hydrogen generation would be problematic.

GE / Caltech Technical Approach:

With the aforementioned technical and design constraints considered, the favored approach was one in which discrete PEC elements are mounted in an inert membrane material. This is shown schematically in Figure 3. The advantages of this type of arrangement are two-fold. First, the architecture allows for relatively inexpensive particulate photo-catalysts to be used instead of more expensive architectures such as thin-films. Furthermore, since a gas-impermeable membrane separates the cathodic and anodic reactions, hydrogen and oxygen generation takes place on opposite sides of the cell, eliminating the need for downstream gas separation. Though this type of architecture may seem to be limited to a single photo-catalyst cell, a dual photo-catalyst (tandem) approach is also feasible. Thus, the materials discovery portion of the program was not limited to n-type or p-type photocatalysts. Furthermore, band alignment with the redox potential of water, though important, did not preclude the usage of materials in tandem arrangements. Each of the three arrangements discussed in the Background Section carry with them unique technical challenges. Consequently, the program was divided into six distinct tasks:

1. Perform materials discovery

1.1. Synthesize $\mathrm{C}$ or $\mathrm{N}$ doped metal oxide materials using combinatorial techniques

1.2. Synthesize nitride and/or carbide main group semiconductor materials using combinatorial techniques

1.3. Create new multi-electron transfer catalysts following a rational synthesis approach

1.4. Develop polymer membrane supports

2. Characterize and test materials discovered

2.1. Characterize and test new photo-anode materials

2.2. Characterize and test new photo-cathode materials

2.3. Characterize and test new electron transfer catalysts

2.4. Characterize and test polymer membrane supports

3. Design "balance of plant" for photo-catalytic solar water splitting system

4. Build solar water-splitting system prototypes using various materials combinations

5. Test and down-select materials combinations in photo-catalytic solar water splitting system

6. Optimize water splitting system to improve efficiency, durability, and cost effectiveness

The overall program workflow as initially proposed is shown in Figure 4. The program Gantt chart is shown in Figure 5. As shown in the Gantt chart, Tasks 1 through 3 was intended to be the focus of research prior to Tollgate 1 . Tollgate 1 was intended as a determination of progress, particularly with respect to materials discovery. A go / no-go decision was anticipated after Tollgate 1, which was expected to require 1 year of coordinated effort. Tollgate 2 was intended as a determination on progress with respect to PEC system-level tasks; tasks 1.4 and 3 through 5 in particular. This tollgate again was expected to require 1 year of R\&D. Tollgate 3 corresponded to program conclusion, where the systems-level performance was to be compared to the DOE program deliverables as captured in the original solicitation.

As the program was discontinued prior to Tollgate 1, the discussion will be limited to research carried out in phase 1 of the program; namely Tasks 1 through 3, (minus subtasks 1.3 and 2.3).

\section{Results}

\section{Task 1: Perform materials discovery}

1.1: Synthesize $\mathrm{C}$ or $\mathrm{N}$ doped metal oxide materials using combinatorial techniques

A novel combinatorial method to print and test thin films of new materials has been developed to quickly and efficiently search for stable photo-catalytic materials capable of evolving $\mathrm{H}_{2}$ gas under visible light illumination. This combinatorial approach relies on a very low-cost commercially available ink jet printer to deposit micro-liter quantitatively controlled mixtures of metal salt solutions. Furthermore, in a 
matter of seconds the ink jet printer is able to print up to 256 spots, each with a unique combination of up to eight different metals, directly onto a conductive glass substrate (fluorine doped tin oxide coated glass, or FTO). Once the mixed metal salt solutions are printed and pyrolyzed by baking in air to form mixed metal oxides, each glass slide is tested under white light illumination in a $\mathrm{NaOH}$ electrolyte solution to screen for photocurrent. The great advantage of this method is the combination of the incredible speed and versatility of the printing and screening process, as well as its simplicity and low cost.

To test the accuracy of the mixtures made by the printer, 252 spots were printed on an FTO slide, with each spot containing a mixture of two metals. This is shown in Figure 6. The composition of each spot was systematically varied by $10 \%$ jumps in elemental ratio (e.g. 10:90::Fe:Co, 20:80::Fe:Co, and so on for each combination of each of the eight metals). The composition of the resulting pyrolyzed metal oxides was determined by both X-ray photoelectron spectroscopy and energy dispersive spectroscopy. The measured elemental ratios agree well with the values set in the customized user-controlled printer driver. It is worth noting that certain metal oxide spots were not uniform, with segregation and cracking observed in the scanning electron microscope images for some elemental combinations. However, the differing morphologies were generally reproducible from slide to slide and are attributed to the inherent stability of the metal oxide phase rather being a function of printing method itself.

1.2: Synthesize nitride and/or carbide main group semiconductor materials using combinatorial techniques

The vast majority of this research in main group nitrides has focused on tantalum-containing species, particularly $\mathrm{TaON}, \mathrm{Ta}_{3} \mathrm{~N}_{5}$ and rare-earth tantalum oxynitrides. $\mathrm{Ta}_{3} \mathrm{~N}_{5}$ was originally synthesized in the 1960 's, but only recently has this material shown demonstrated photodecomposition of water ${ }^{5}$. The rareearth oxynitride materials discussed here typically form perovskite structures of the general form $\mathrm{ABO}_{2} \mathrm{~N}$, with $\mathrm{Ba}^{2+}, \mathrm{Sr}^{2+}$, or $\mathrm{Ca}^{2+}$ on the A-site, and $\mathrm{Ta}^{5+}$ on the $\mathrm{B}$-site ${ }^{6}$. Nitrogen and oxygen are interchangeable, thus a disordered oxynitride forms. They vary in color from yellow to brown, depending on the rare-earth species. Due to the inherent difficulty in making bulk samples or thin-films, the literature regarding photodecomposition of water using rare-earth tantalum oxynitrides is incomplete.

Since tantalum is a multivalent cation, both tantalum (III) nitride and tantalum (V) nitride are stable phases. In this case, the +5 oxidation state is the desired phase. Consequently, we found it beneficial to begin with tantalum (V) oxide as a starting material. Solid-state nitridation of the respective oxides (or mixtures thereof) was used to synthesize $\mathrm{TaON}, \mathrm{Ta}_{3} \mathrm{~N}_{5}$ and rare-earth tantalum oxynitrides. This was carried out at high temperatures in an ammonia-rich atmosphere. Nitridation temperatures vary from $800^{\circ} \mathrm{C}$ to $900^{\circ} \mathrm{C}$, and total nitridation time varied from 24-72 hours. Examples are shown in Figure 7. Phase purity generally increased with nitridation time. To better facilitate homogeneity, the powders were cooled and milled in 2-propanol prior to re-fire in 12-hour increments. Despite multiple firings, a trace amount of $\mathrm{Ta}_{3} \mathrm{~N}_{5}$ was found in the rare-earth oxynitrides. However, this amount was not sufficient to interfere with the characterization of these materials.

$\mathrm{Ta}_{3} \mathrm{~N}_{5}$ and rare-earth tantalum oxynitrides were also prepared in thin-film form. As is the case for powder-based samples, the key to synthesis is pre-stabilizing the $\mathrm{Ta}^{5+}$ oxidation state, which is conveniently done by starting with the oxide (rather than elemental Ta). The preparation procedure is as follows:

- Deposition of a precious metal (i.e. Pt) back contact to an alumina substrate.

- Deposition of an elemental tantalum layer over the platinum back contact.

- Oxidation of elemental tantalum at $1000^{\circ} \mathrm{C}$ in flowing oxygen.

- Nitridation in an $\mathrm{NH}_{3}$ atmosphere at $800-1100^{\circ} \mathrm{C}$ from $24-72$ hours (depending on film thickness).

A $\mathrm{Ta}_{3} \mathrm{~N}_{5}$ film made using this technique is shown in Figure 8. This process was also used to produce compositionally graded thin-films for compositional optimization studies. An example of such a film is shown in Figure 9. These films will be discussed in greater detail in Task 2.

\section{4: Develop polymer membrane supports}

As discussed earlier, a polymeric membrane to carry the scaffolds is envisaged. This overall concept is not unique. Similar techniques were proposed for applications such as PV and batteries ${ }^{7,8}$. To test the

\footnotetext{
${ }^{5}$ Hara et al, J. Phys. Chem. B, 107 (2003) 13441-13445

${ }^{6}$ Marchand et al, J. European Cer. Soc., 8 (1991) 197-213

${ }^{7}$ US Patent 3,736,476

${ }^{8}$ US Patent 4,247,499
} 
overall efficacy of the technique, large-grained alumina powder $(120-150 \mu \mathrm{m})$ was loaded into a polypropylene membrane as described in ref [8]. An optical micrograph of the resultant film is shown in Figure 10.

\section{Task 2: Characterize and test materials discovered}

2.1, 2.2: Characterize and test new photo-anode / photo-cathode materials:

Though separated at the subtask level, the characterization techniques are generally the same for photo-anode and photo-cathode materials. The principal difference would be the gaseous species generated at the semiconductor / electrolyte interface. In addition, as discussed in the Background section, the counter electrode, and perhaps the electrolyte, could differ as well. These differences, where applicable, will be called out specifically. Bulk specimens and combinatorial chemistry-derived specimens required individualized characterization techniques. Furthermore, Caltech and GE had specific testing capabilities designed primarily to suit the form factor of the as-generated sample sets. Caltech's characterization efforts, which were designed to handle large sample sets simultaneously, will be discussed separately from GE's efforts, which were designed to handle bulk powders and compositionally-graded film specimens.

Characterization of combinatorial chemistry-based discrete sample sets:

To test for photo-catalytic activity, a computer-controlled translation stage was used to raster the FTO slide in front of a beam of white light focused down to the size of single metal oxide spot. Using a platinum counter electrode and a de-oxygenated aqueous solution of $1 \mathrm{M} \mathrm{NaOH}$, the short-circuit current was measured for each spot individually. By chopping the light and using a lock-in amplifier to analyze the photocurrent, it is also possible to measure photocurrents when biasing the sample without any contribution from the dark current. Using a custom automated scanning and data collection program, a FTO slide with over 250 unique materials on it can be screened in less than 30 minutes. For easy interpretation, the data was plotted in a false color image, with the photocurrent plotted versus position on the slide. This is shown in Figure 11. Positive or negative currents indicate strong photo-anodic or photo-cathodic behavior respectively.

Having developed a robust method for printing and testing materials for photo-catalytic activity, we have tested a number of combinations metals, and several combinations appear promising. We have also observed that for some combinations of metals, the measured photocurrents are not constant with time, indicating possible degradation or corrosion of the metal oxide film under illumination. We have established a protocol and shown that we can print and screen at a rate to support a throughput of approximately 2,000 materials per day, and have initiated an extensive parallel searching approach at present to apply this technology to new photo-electrode material discovery.

\section{Characterization of nitride-based powder samples:}

Diffuse reflectance was used to estimate the band gap of nitride and oxynitride powder samples. A Spex Fluorolog 3 with a Xe arc lamp was used to measure the reflectance of samples compared to a $\mathrm{BaSO}_{4}$ reflectance standard. The data was converted to absorbance using a Kubelka-Monk transform. This data is shown in Figure 12.

The location of the valence and conduction bands compared to the vacuum level was measured via UPS as taught by Chun et $\mathrm{al}^{9}$. These results are summarized in Figure 13. In addition to the rare-earth oxynitrides and tantalum nitride, $\mathrm{SrTiO}_{3}$ was measured as a reference. The measured valence-band maximum of $7.65 \mathrm{eV}$ for $\mathrm{SrTiO}_{3}$ is comparable to that reported in the literature ${ }^{10}$. In contrast to that reported by Chun et al, specimen charging was an issue. This is especially evident in the $\mathrm{SrTaO}_{2} \mathrm{~N}$ specimen, which exhibited a valence band shifted roughly $1 / 0 \mathrm{eV}$ lower in energy compared to the other specimens. Consequently, This data is only to serve as a guide in photocatalyst development, and improved sample quality will be necessary for proper characterization.

\section{Characterization of combinatorial chemistry-based films:}

As discussed in Task 1.2, the goal was to test compositionally-graded thin-film specimens so as to understand the photoelectrochemical performance as a function of composition, particularly for solidsolutions of the form $(\mathrm{Ca}, \mathrm{Sr}, \mathrm{Ba}) \mathrm{TaO}_{2} \mathrm{~N}$. Consequently, development of this capability was evolutionary,

${ }^{9}$ Chun et al, J. Phys. Chem. B, 107 (2003) 1798-1803

${ }^{10} \mathrm{Xu}$ et al, Amer. Mineralogist, 85, (2000) 543-556 
beginning with the development of suitable test apparatus and sample geometry. $\mathrm{Ta}_{3} \mathrm{~N}_{5}$ thin-films were tested in a nitrogen-sparged $\mathrm{KOH}$ solution. Preliminary results are shown in Figure 14. As seen from the data, the sample is indeed photoelectrochemically active (no external bias applied). Significant improvements were also made to our ability to characterize photocatalysts. Specifically, we constructed a laboratory-scale system for evaluating performance of photocatalysts that includes gas chromatography analysis of $\mathrm{H}_{2}$ production. This is the Gen III cell shown in Figure 13. From this, we can measure both evolved hydrogen and electronic transport in situ. This will allow us to make real-time measurements of efficiency as well as photodegradation effects.

\section{Discussion}

The general focus of the program was to develop stable photo-catalysts suitable for solar water splitting. The systems-level efforts were intended to help frame the technical problem and provide insight as to the desired photo-catalyst (composition, form factor, cost, etc.). Based on a design utilizing discrete photo-catalysts embedded in a polymeric membrane, the following materials constraints can be surmised:

1. Membrane thickness has a considerable impact on system performance.

2. For non-oxide photo-catalysts, having the water oxidation step take place on the photocatalyst surface offers significant potential for photo-oxidation.

3. From a manufacturing perspective, a single photo-catalyst system is significantly less complex. However this puts considerable performance constraints on the photo-catalyst.

4. From a cost perspective, the PEC module cost should be comparable to a conventional PV module. Since PV modules are typically amortized over 20 years, and the performance lifetime target for PEC modules is 5000 hrs, the photo-catalyst membrane combination will need to be replaceable without adding significant cost to the module.

The results presented in the previous section will be discussed with respect to the aforementioned constraints.

\section{Perform materials discovery:}

The materials discovery activities took two parallel paths. The first, led by the team at Caltech, focused primarily on oxide-based photo-catalyst discovery. This activity was a more general materials screen, thus it is understandable that over the given funding period, a particular photo-catalyst meeting the desired constraints was not discovered. However, the development of a robust set of screening tools is important, and hopefully will benefit subsequent programs in PEC technology. Combinatorial chemistry remains the best approach to screen relatively disparate regions of materials space. However, combinatorial chemistry is suitable only as a qualitative screening tool. As with most electronic materials, means of synthesis, purity, etc can have a pronounced effect on outcome. Consequently, the capabilities developed in this program are by themselves incomplete; further tools are needed, particularly with respect to rapid phase analyses (to complement combinatorial samples). Furthermore, some predictive tool should be coupled to output from combinatorial studies. This would involve modeling capabilities outside the purview of this program. Better understanding of structure-property relationships as they relate to photoelectrochemical processes is lacking.

The second path, led through GE, was more focused on a specific non-oxide (and oxynitride) materials space. Here the combinatorial tools were used to make perturbations around a specific set of compositions. Thus, progress in this approach was more evident. The results from this research can be summarized as follows:

1. Nitrides and oxynitrides based on tantalum in the +5 valence state were studied.

2. Of this general class of materials, alkaline-earth tantalum oxynitride perovskites of the general form $\mathrm{ATaO}_{2} \mathrm{~N}$ show promise

3. Compared to oxides, the significant shift in the valence band energy level can be attributed to hybridization of nitrogen-oxygen $2 p$ orbitals.

4. It was found that by changing the alkaline-earth species, the ligand-field strength within these perovskite oxynitrides could be perturbed such that the conduction band can be shifted with respect to the vacuum level.

As shown in Figure 15, the valence and conduction bands of nitride-based photo-catalysts are better positioned with respect to the redox potentials of water than their oxide counterparts. Furthermore, it can be concluded that the provskites of general form $\mathrm{ABO}_{2} \mathrm{~N}$ can be tuned to better align with the reduction band of $\mathrm{H}^{+}$. Though important, band gap alignment by itself is not sufficient for efficient water splitting. 
Electronic performance, particularly with respect to minority carrier diffusion length, is also important. In order to carry out research and development of this family of photo-catalysts, a thin-film approach makes short-term sense due to the ability to characterize films compared to conventional bulk powders.

Consequently, development of thin-film specimens for electronic characterization became the focus of development efforts. Of course, the options to process tantalum-based thin films are rather limited. For this reason, sputtering was chosen as the film processing technique.

In order to develop the characterization protocol first, $\mathrm{Ta}_{3} \mathrm{~N}_{5}$ was chosen as the test material. The reasoning was threefold: First, $\mathrm{Ta}_{3} \mathrm{~N}_{5}$ is not susceptible to compositional variability (since only one element is being deposited). Second, processing of $\mathrm{Ta}_{3} \mathrm{~N}_{5}$ was already optimized on bulk powders. Third, there is some literature regarding the electrochemical performance for comparison. Figure 8 shows an example of the film resulting from the procedure outlined in the Results section. Based on the wine-red color of the film, it was concluded that the film was indeed $\mathrm{Ta}_{3} \mathrm{~N}_{5}$. The program ended before a more comprehensive compositional analysis could be conducted. As mentioned in the Results section, the groundwork for investigating alkaline-earth tantalum oxynitrides was also performed. Figure 9 shows a Ca-Ta simultaneous deposition. Characterization of these films will be discussed in succeeding paragraphs.

\section{Characterize and test materials discovered:}

Thin film deposition of samples suitable for electrochemical characterization was limited to $\mathrm{Ta}_{3} \mathrm{~N}_{5}$. In retrospect, this material was probably not the ideal candidate due to the relatively low cathodic overpotential. Furthermore, the deposition technique was not optimized for electronic performance. Nonetheless, the sample did show photoelectrochemical activity, albeit at relatively low quantum efficiency. There are many plausible explanations for why the performance is quite low, though the most valid reason is that this was a first test of non-optimized equipment on a non-optimized specimen. With more time to better diagnose the specimens, it is expected that the performance would improve dramatically. The purpose of these efforts was to develop a testing method; therefore outputs such as backcontact stability, hydrogen/oxygen measurement, etc. Figure 13c shows the finalized cell design. The overall design evolved due to difficulties experienced with prior designs. Common difficulties that had arisen include:

1. Sample edge effects

2. Photo-induced corrosion of electrical connections

3. Compatibility with solar-simulator equipment

4. Compatibility with gas-chromatography (GC) equipment

The finalized cell design addressed each of these issues. By moving both the edges and contacts outside of the electrolyte (cell "sandwiched" the substrate), both edge effects and electrical connection problems were eliminated. The reduced overall size of the cell afforded greater compatibility with our existing solarsimulator equipment. Finally, gas connections allowed simultaneous GC and I-V measurements.

The program was canceled prior to optimization of the test equipment. Thus, correlated GC and I-V measurements were not performed. Delays, particularly in the calibrated light source, hindered progress.

\section{Conclusions}

From the perspective of technology feasibility, there is enough data to conclude that photoelectrochemical processes offer the potential to provide an inexpensive, abundant source of hydrogen while generating nearly zero greenhouse gas emissions. However, the commercial feasibility is much more difficult to assess, largely due to the fact that so much is unclear regarding how hydrogen will be generated, transported, and stored. Clearly, the principal benefit of photoelectrochemical processes is the fact that the energy used to drive the process originates from the sun. The question then becomes how the hydrogen value chain evolves. Assuming cost-of-electricity trends remain consistent, it's difficult to imagine scenarios where photoelectrochemical hydrogen achieves a significantly reduced cost position compared to technology like coal gasification or electrolysis. However, the fact that photoelectrochemical hydrogen can be implemented without a robust hydrogen transport infrastructure adds appeal. Furthermore, it's difficult to project the eventual role greenhouse gas emissions will play selection of our next transportation fuel.

Ultimately, there is still much work that needs to be done to assess the technical and commercial viability of this technology. Though still early, certainly there is ample evidence that photoelectrochemical processes merit additional study. In particular, the perovskite-based tantalum oxynitrides studied in this program offer the potential of retiring one of the key milestones in photoelectrochemical development; that being the photocatalyst. It can be concluded from this study that p-type photo-cathode particles with a thin 
metal (platinum at first, lower-cost materials later) coating on the anodic side of the membrane could potentially meet long-term DOE goals for hydrogen cost via photoelectrochemical processes. However, evidence that the perovskites studied here can meet these requirements is scant. Much work, specifically with regard to electronic properties, is required. In order to meet these performance goals, the electronic properties, particularly minority carrier transport, would have to rival that of silicon. Thus, a long-term development strategy is appropriate; which is consistent with the DOE position on photoelectrochemical hydrogen development. 


\begin{tabular}{|l|c|c|}
\hline Location & $\begin{array}{c}\text { Average insolation in } \\
\text { Jan / Jul (W/m²/hr) }\end{array}$ & $\begin{array}{c}\text { Production rate* } \\
\text { kg/day }\end{array}$ \\
\hline Anchorage & $42 / 192$ & $247.5 / 1132$ \\
\hline Albany & $112.5 / 254$ & $634 / 1500$ \\
\hline L.A. & $195 / 296$ & $1148 / 1748$ \\
\hline Phoenix & $229 / 317$ & $1369 / 1898$ \\
\hline Honolulu & $221 / 275$ & $1305 / 1620$ \\
\hline Singapore & $185 / 188$ & $1091 / 1110$ \\
\hline
\end{tabular}

Table 1: Seasonal generation of hydrogen for various global locations. 


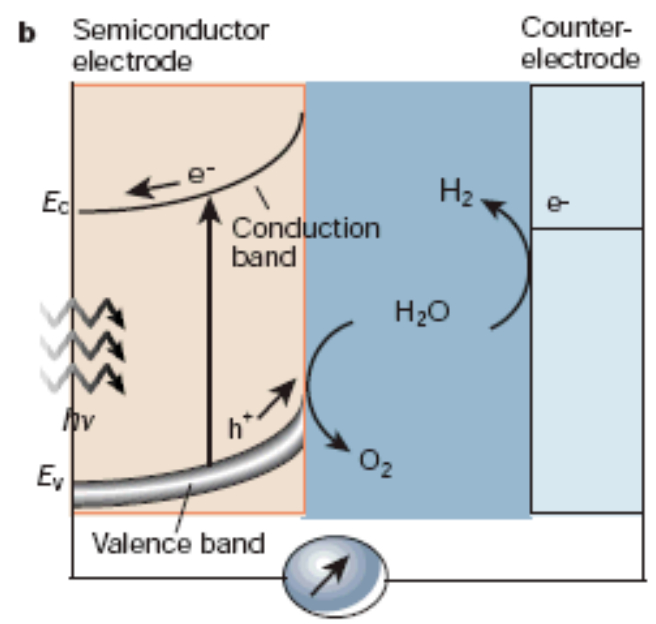

Figure 1: Simplified reaction scheme for photoelectrochemical hydrogen production (Ref 2)

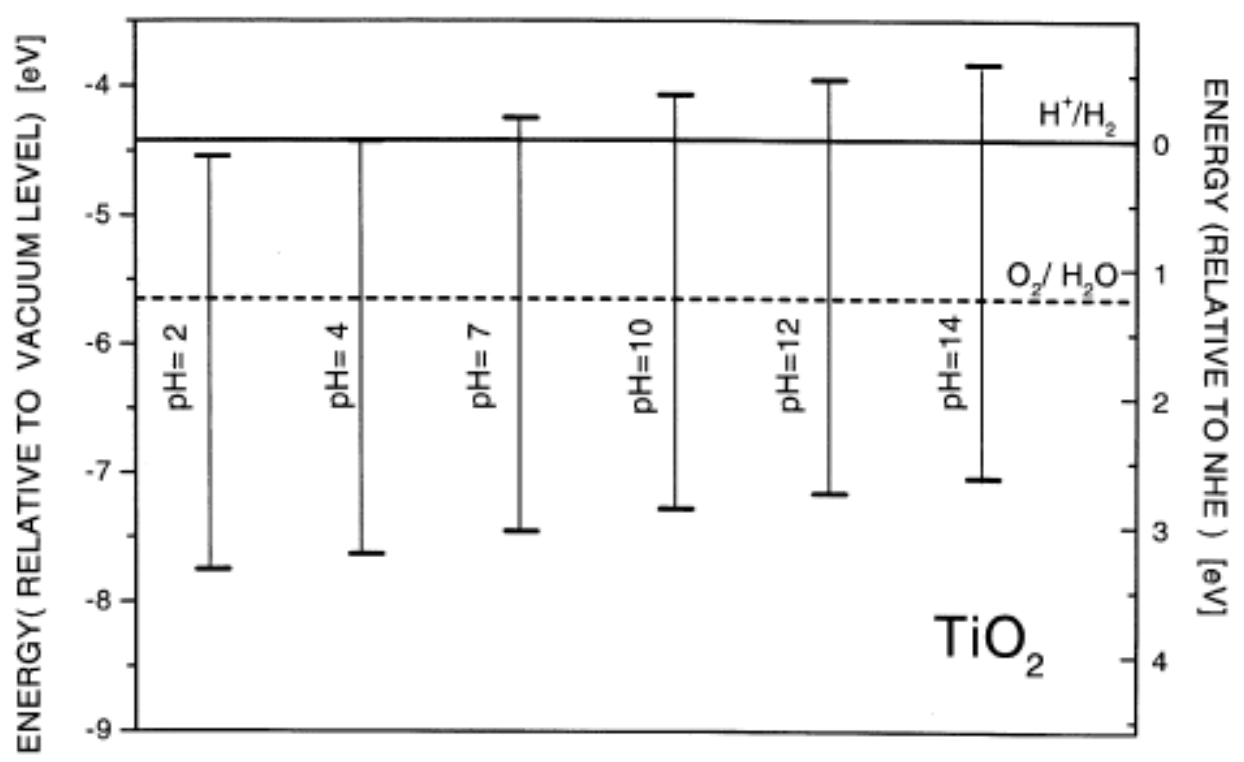

Figure 2: Dependence of band location on $\mathrm{pH}$ of electrolyte (Ref 4) 


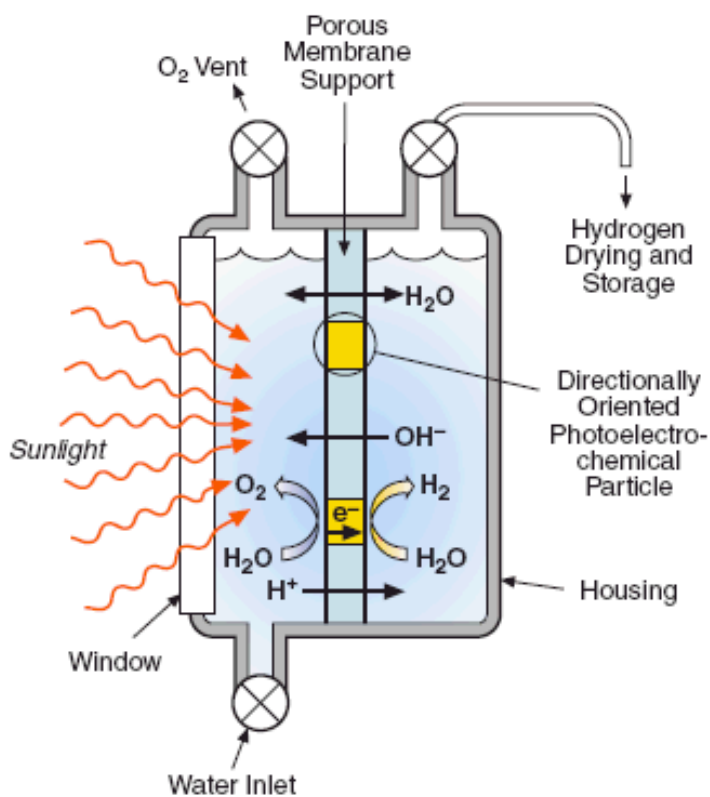

Figure 3: Proposed photoelectrochemical cell design.

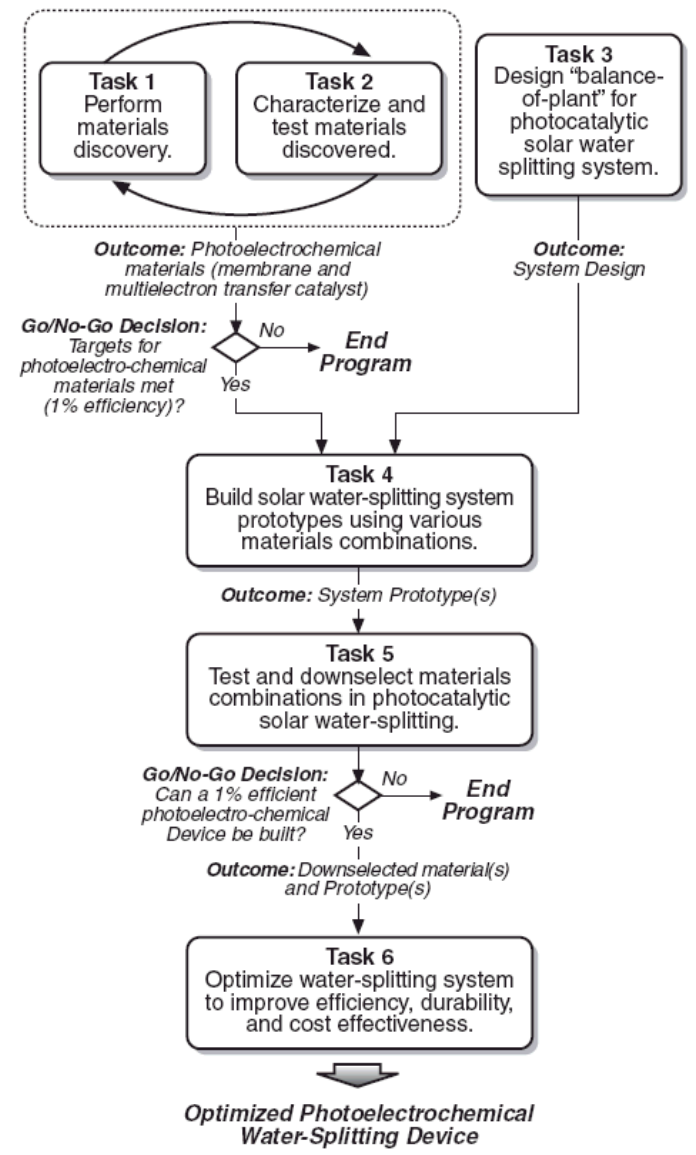

Figure 4: Program workflow diagram. 
Task 1 Perform materials discovery.

1.1 Synthesize $\mathrm{C}$ or $\mathrm{N}$ doped metal oxide materials using combinatorial techniques.

1.2 Synthesize nitride and/or carbide main group semiconductor materials using combinatorial techniques.

1.3 Create new multielectron transfer catalysts following a rational synthesis approach.

1.4 Develop polymer membrane supports.

Task 2 Characterize and test materials discovered.

2.1 Characterize and test new $\mathrm{C}$ or

$\mathrm{N}$ doped metal oxide materials.

- High-throughput screening.

- Fundamental studies.

2.2 Characterize and test new nitride and/or carbide main group semiconductor materials.

- High-throughput screening.

- Fundamental studies.

2.3 Characterize and test new electron transfer catalysts.

2.4 Characterize and test polymer membrane supports.

Task 3 Design "balance-of-plant" for photocatalytic solar water splitting system.

Task 4 Build solar water-splitting system prototypes using various materials combinations.

- Polymer membranes.

- Alumina membranes

Task 5 Test and downselect materials combinations in photocatalytic solar water-splitting.

- Polymer membranes.

- Alumina membranes

Task 6 Optimize water-splitting system to improve efficiency, durability, and cost effectiveness.

Legend: $\nabla$ Program Go/No-Go • Task Go/No-Go
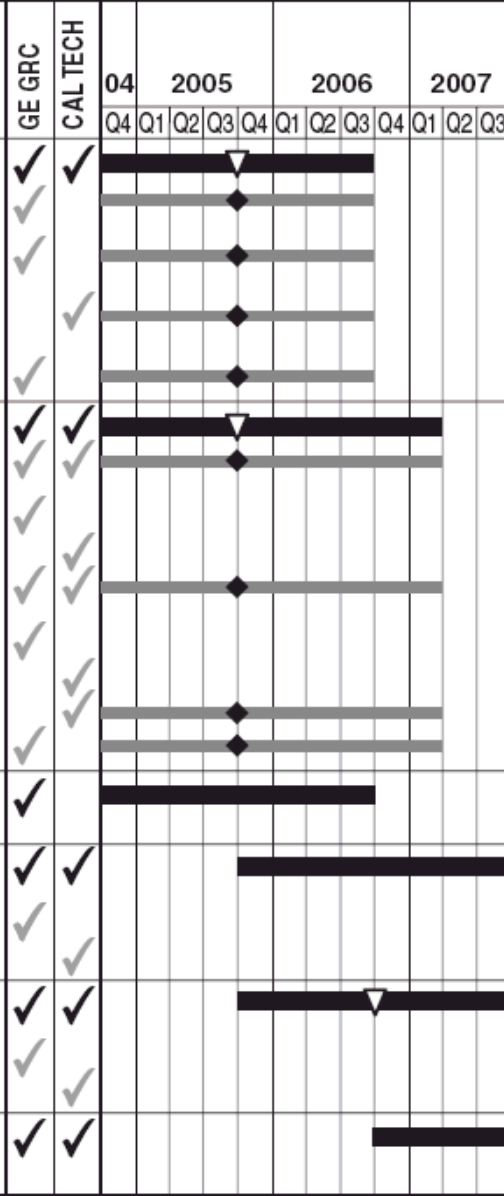

Figure 5: Program Gantt chart

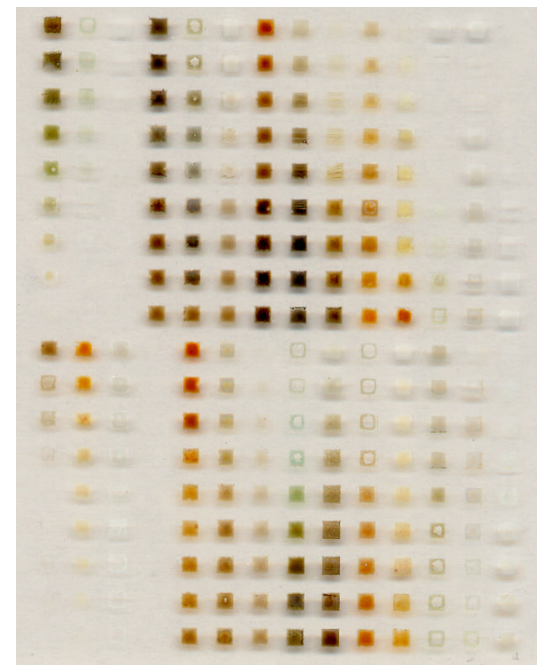

Figure 6: FTO slide printed with binary combinations of eight metals 


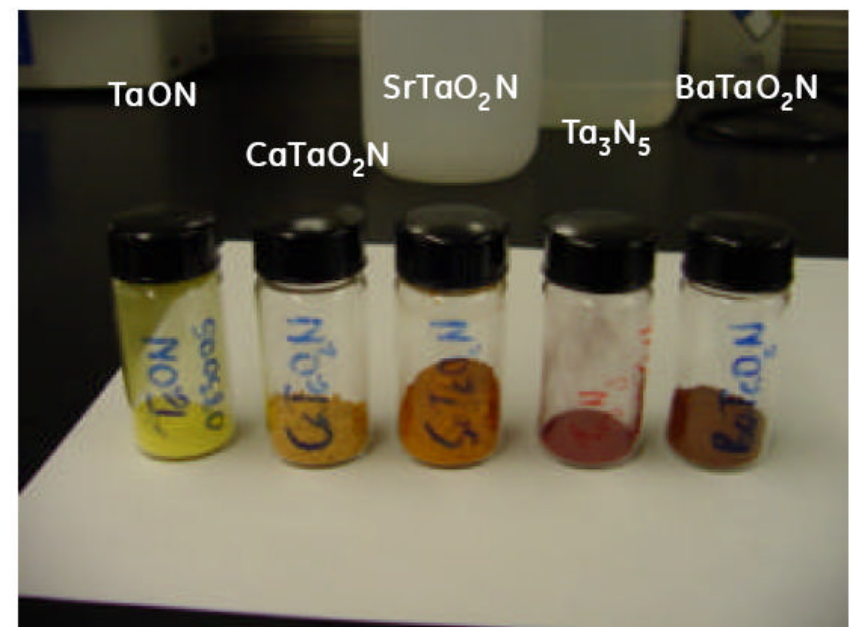

Figure 7: Tantalum-based nitride and oxynitride powders

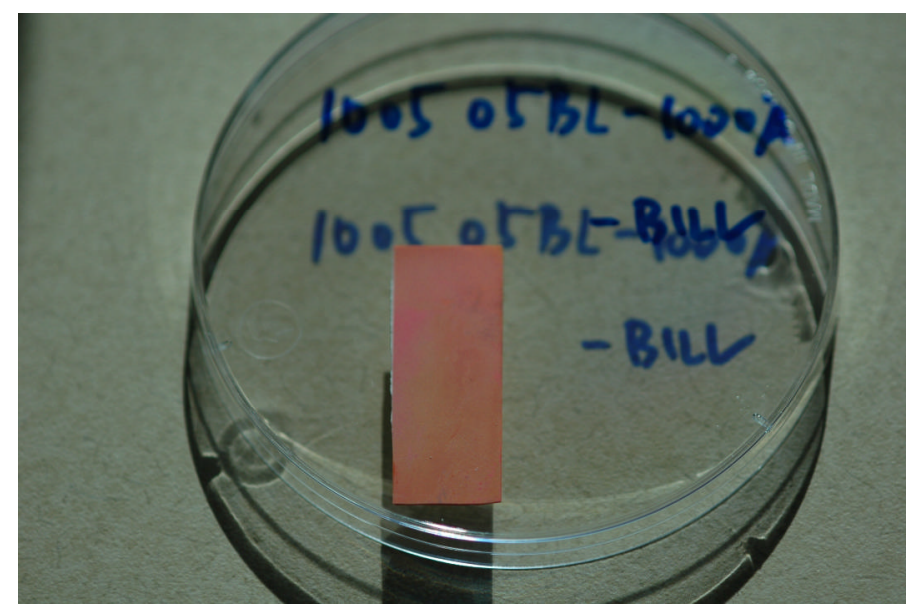

Figure 8: $\mathrm{Ta}_{3} \mathrm{~N}_{5}$ thin film sputtered on platinum-coated alumina substrate 


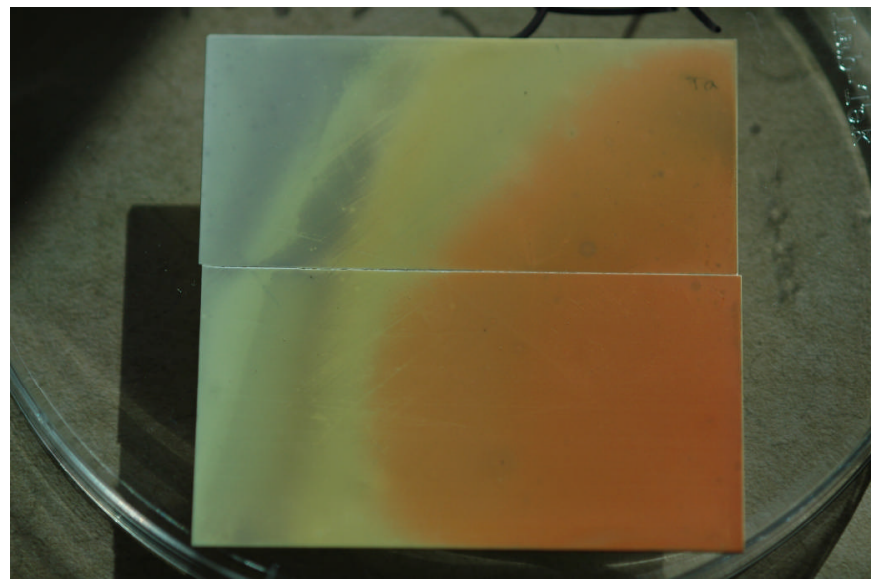

Figure 9: Compositionally-graded Ca-Ta thin film (nitrided)

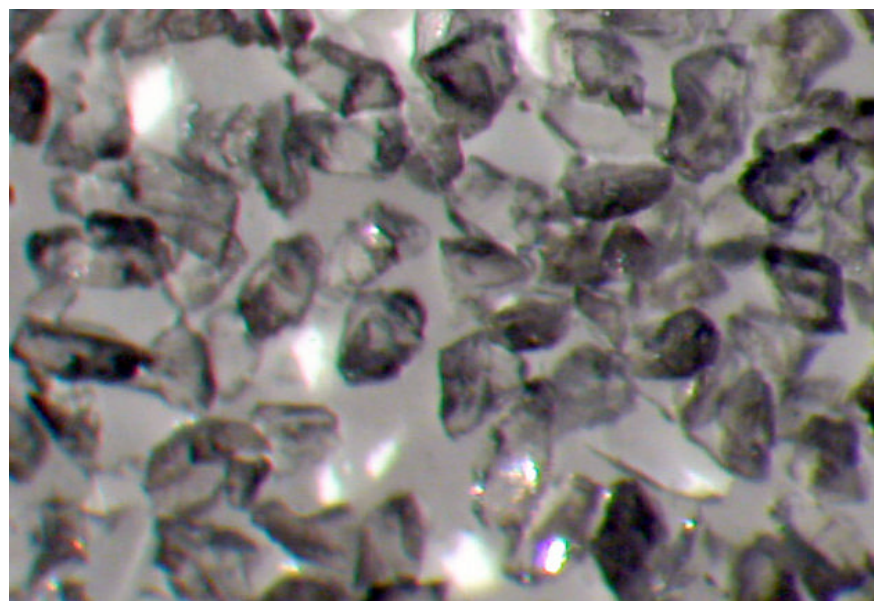

Figure 10: Polyethylene film loaded with $120-150 \mu \mathrm{m}$ alumina particles 


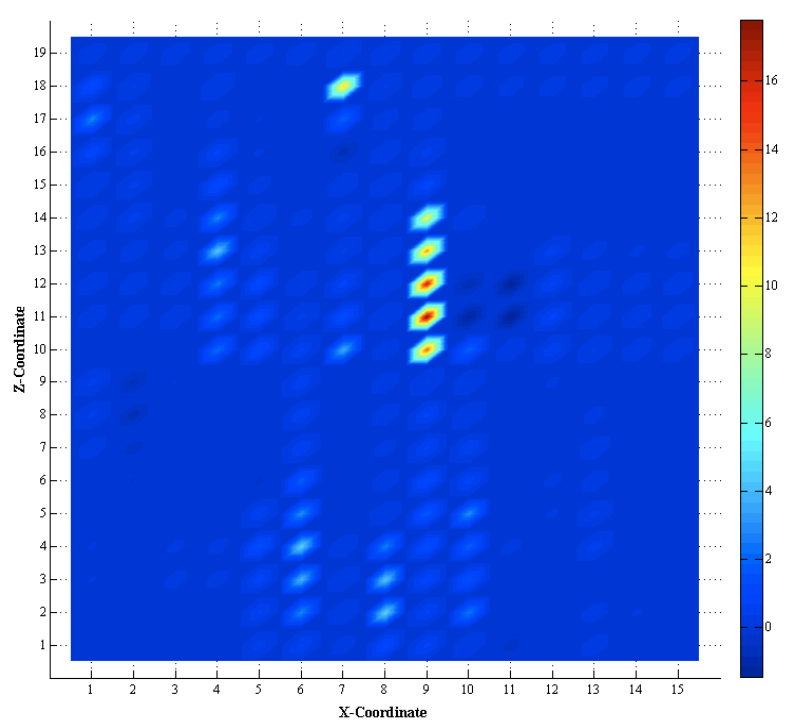

Figure 11: Photocurrent map of a slide of metal oxides

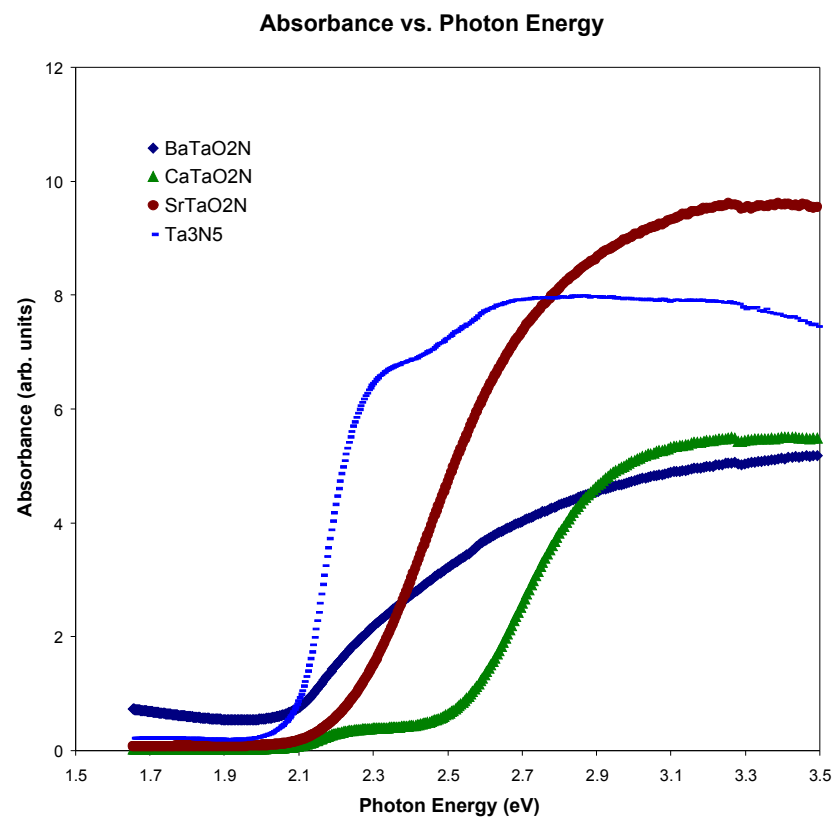

Figure 12: Absorbance versus photon energy for tantalum-based photo-catalysts 


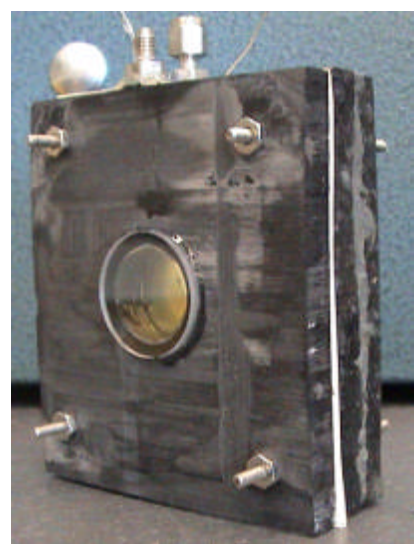

a.

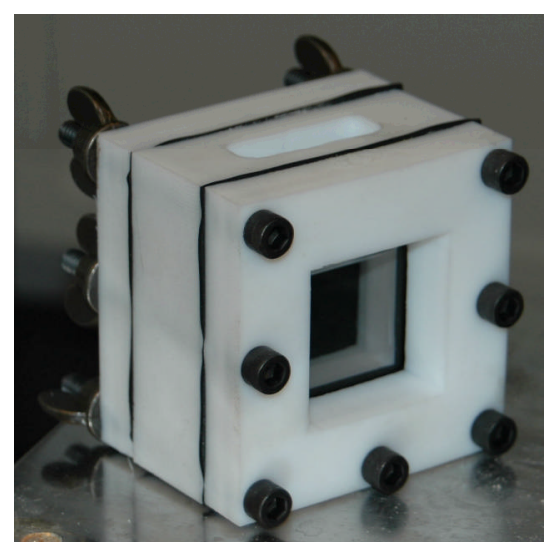

b.

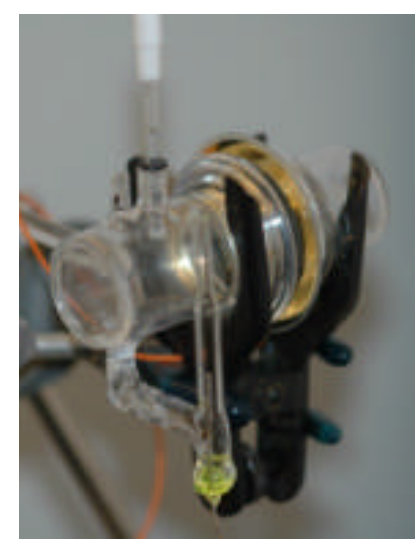

c.

Figure 13: a) Gen I, b) Gen II, c) Gen III photoelectrochemical test cells

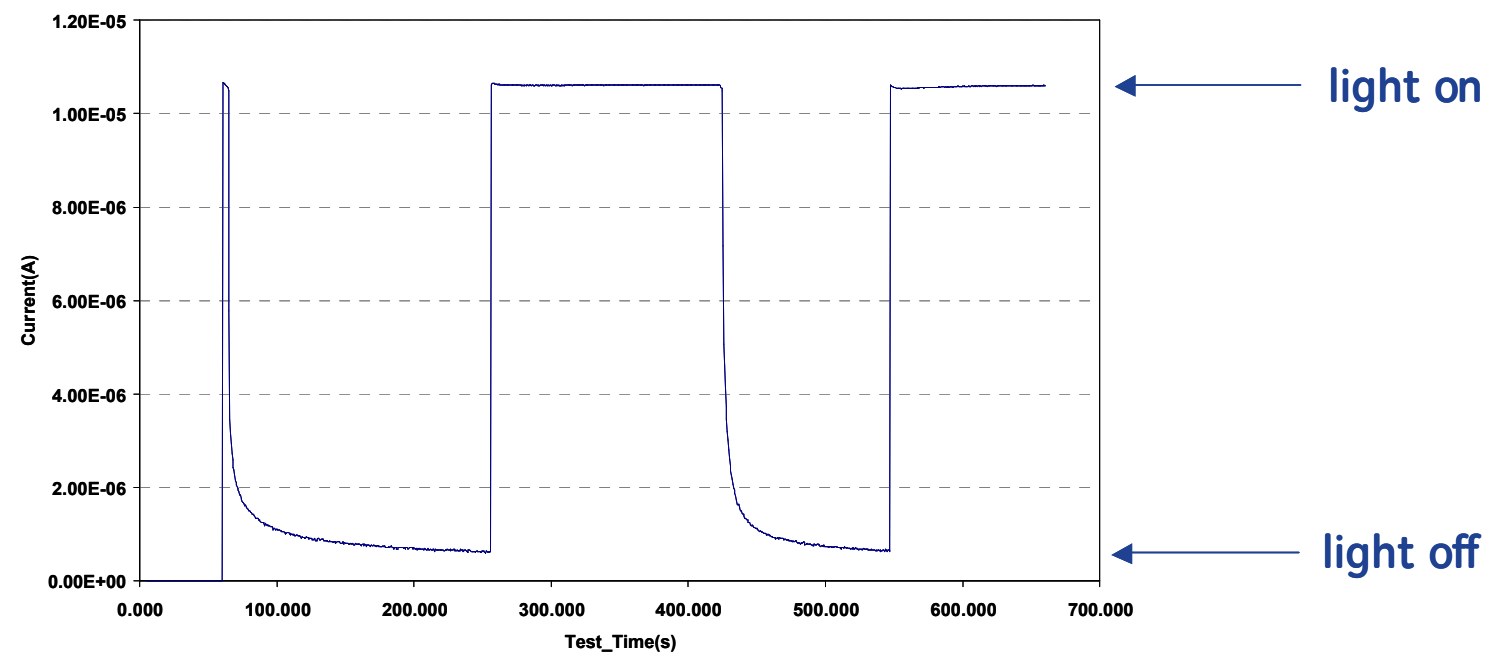

Figure 14: Photo-current vs. time for $\mathrm{Ta}_{3} \mathrm{~N}_{5}$ film in $1 \mathrm{M} \mathrm{NaOH}$ 


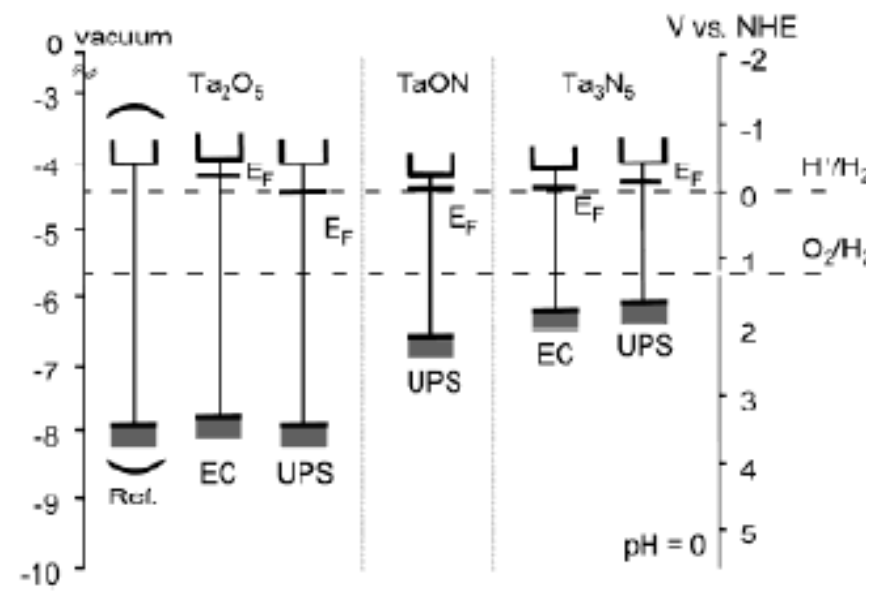

Figure 15: Band level diagram for tantalum-based oxide, oxynitride, and nitride species (Ref 5) 